Supporting Information

\title{
On the Role of Torsional Flexibility in the Film Formation Process in Two $\pi$-Conjugated Model Oligomers
}

Stefan Wedler ${ }^{1}$, Cheng Zhou ${ }^{2}$, Gulllermo C. Bazan ${ }^{2}$, Fabian PANZER $^{1}$, AND ANNA KÖHLER ${ }^{* 1,3}$

${ }^{1}$ Soft Matter Optoelectronics, Experimentalphysik II, University of Bayreuth, Bayreuth 95440, Germany

${ }^{2}$ Departments of Chemistry and Chemical Engineering, National University of Singapore, Singapore, 119077

${ }^{3}$ Bayreuth Institute of Macromolecular Research (BIMF) and Bavarian Polymer Institute (BPI), University of Bayreuth, 95440 Bayreuth, Germany

${ }^{*}$ mail: Anna.Koehler@uni-bayreuth.de 


\section{Experimental Methods}

\subsection{Sample preparation}

The molecules 7,7'-(3,3'-dihexyl-[2,2'-bithiophene]-5,5'-diyl)bis(6-fluoro-4-(5-hexylthiophen-2-yl)benzo[c] [1,2,5]thiadiazole) (TT) and 7,7'-(4,4-dihexyl-4H-cyclopenta[2,1-b:3,4-b']dithiophene-2,6-diyl)bis(6fluoro-4-(5-hexylthiophen-2-yl)benzo[c][1,2,5]thiadiazole) (CT) were synthesized as reported previously, 1 their structures are shown in Figure 1 in the main manuscript. Solutions for spin coating were prepared by dissolving each molecule in hexane to a final concentration of $5 \mathrm{mg} \mathrm{ml}^{-1}$ for CT and $4 \mathrm{mg} \mathrm{ml}^{-1}$ for TT. The solutions were stirred at $150 \mathrm{rpm}$ and heated to $50^{\circ} \mathrm{C}$ for $30 \mathrm{~min}$ to ensure solvation prior to spin coating. For spin coating, $20 \mu$ of the prepared solution was dropped onto a round Spectrosil B substrate (diameter: $13 \mathrm{~mm}$ ) and the spin coater was set to $500 \mathrm{rpm}$ for the complete measurement time. It needs $1.0 \mathrm{~s}$ to reach the final rotation speed. The substrate temperature was set to room temperate for $\mathrm{CT}$ and to $51^{\circ} \mathrm{C}$ for $\mathrm{TT}$, which is above the critical temperature for aggregation in both cases as detailed in section 2. For spectroscopic measurements in hexane solution, we used a final concentration of $0.05 \mathrm{mg} \mathrm{ml}^{-1}$ for $\mathrm{CT}$ and put the solution in a quartz cuvette with $1.00 \mathrm{~mm}$ path length.

\subsection{In-situ spectroscopy}

Transmission measurements during film formation were performed using a white LED which is placed under the rotating chuck of the spin coater with a bore along the rotation axis. For emission measurements, a $520 \mathrm{~nm}$ diode laser was used as excitation source. Both, emission from the sample and transmitted white light, was collected via an optical fiber and guided to the home-built detection system. Absorption and emission measurements were performed alternatingly after each detection frame. The detection system consists of a chopper wheel with mirrored blades, which creates two independent beam paths for transmission and emission measurements. An optical longpass filter (550 nm cut-off wavelength) was placed in the beam path for emission measurements to remove residual laser light. Both transmission and emission spectra were recorded with the same spectrograph consisting of a CCD camera (Andor iDus) coupled to a monochromator (Oriel). Full technical details can be found in reference ${ }^{2}$. The temporal origin is defined as the start of the rotation of the spin coater. Minor oscillations in the absorption spectra originate from an aliasing effect between the rotation of the spin coater and the sampling frequency for acquisition. This aliasing effect was suppressed by smoothing the discrete Fourier transform in the vicinity of the aliasing frequency (see section 5). All emission spectra are corrected for the transmission of all optical elements as well as changes in absorption at the laser wavelength. The relative photoluminescence quantum yield (PLQY) was determined by integrating the corrected emission intensity for each time step. Comparing the relative PLQY at $t=0 \mathrm{~s}$ with the PLQY value of a dilute solution yields the absolute PLQY during processing, as all material on the substrate is in solution at that stage. The fraction of aggregates (FoA) was calculated from the absorption spectra by decomposing each spectrum into amorphous and aggregated contributions and comparing their spectral areas after correcting for changes in oscillator strength, as detailed in section 6 . Color codes developed by Fabio $\mathrm{Crameri}^{3}$ were used in $2 \mathrm{D}$ heat maps to prevent visual distortion of the data. 


\section{Critical temperature for aggregation at high concentrations}
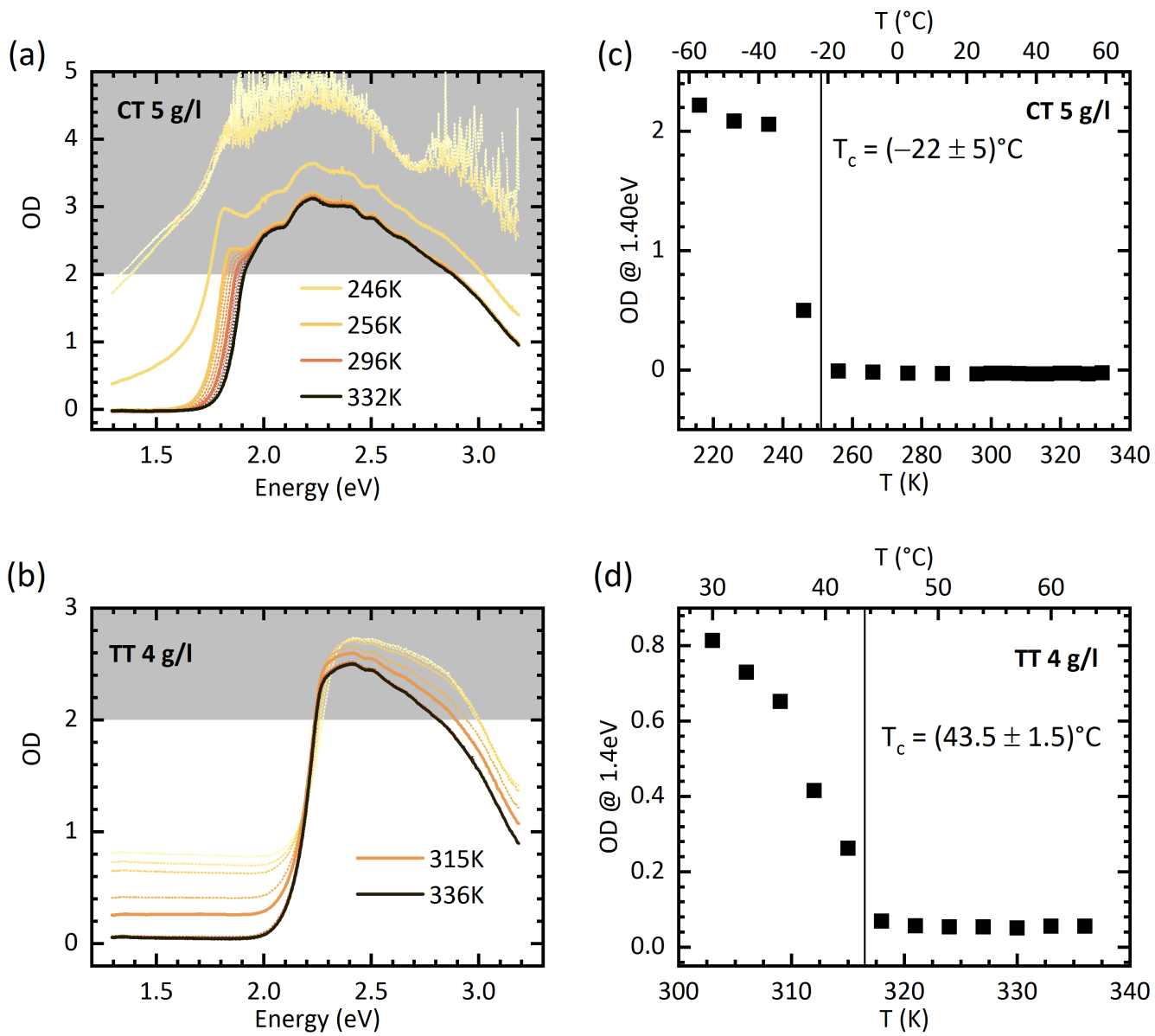

Figure S1: Absorbance spectra for (a) CT and (b) TT in hexane at high concentrations. The spectra were recorded using a quartz glass cuvette with a path length of $1.00 \mathrm{~mm}$ and a homebuilt setup. ${ }^{4}$ For $\mathrm{CT}$, the spectra were recorded in steps of $4 \mathrm{~K}(10 \mathrm{~K})$ above (below) $296 \mathrm{~K}$, for TT the spectra were measured in steps of $3 \mathrm{~K}$. The gray region corresponds to optical density values, where the detector saturates. The evolution of the optical density at the energy of $1.40 \mathrm{eV}$ as a function of temperature is shown in (c) for CT and (d) for TT. It is a measure for scattering and is used for determining the critical temperature for the disorder-order-transition. 


\section{Absorption of CT during spin casting without baseline}

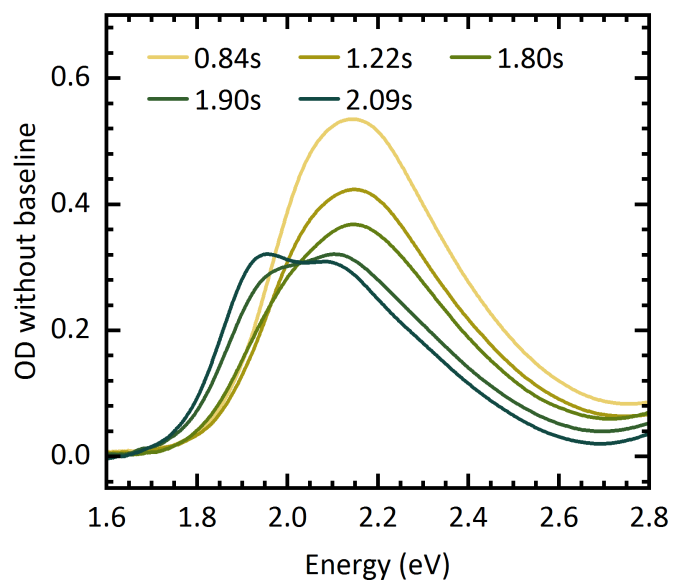

Figure S2: Absorption spectra of CT film during processing (Figure 2b from main manuscript) after subtracting a constant baseline. The isosbestic point is still present, but shifted to $2.03 \mathrm{eV}$.

\section{Full absorption and emission of CT during spin casting}

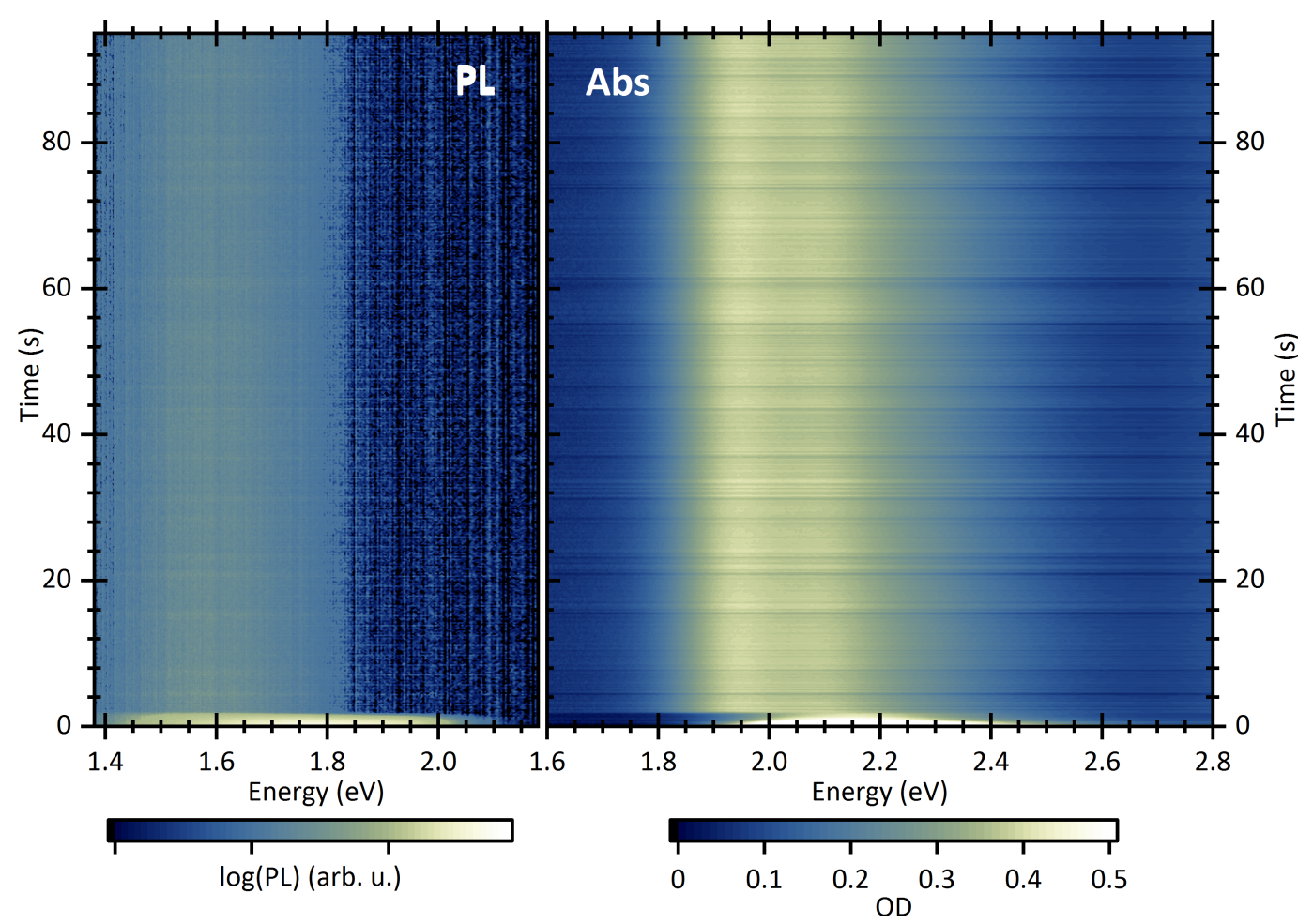

Figure S3: Absorption and emission of the CT film during spin casting for processing times up to $96 \mathrm{~s}$. 


\section{Suppression of the aliasing effect for absorption during spin casting}
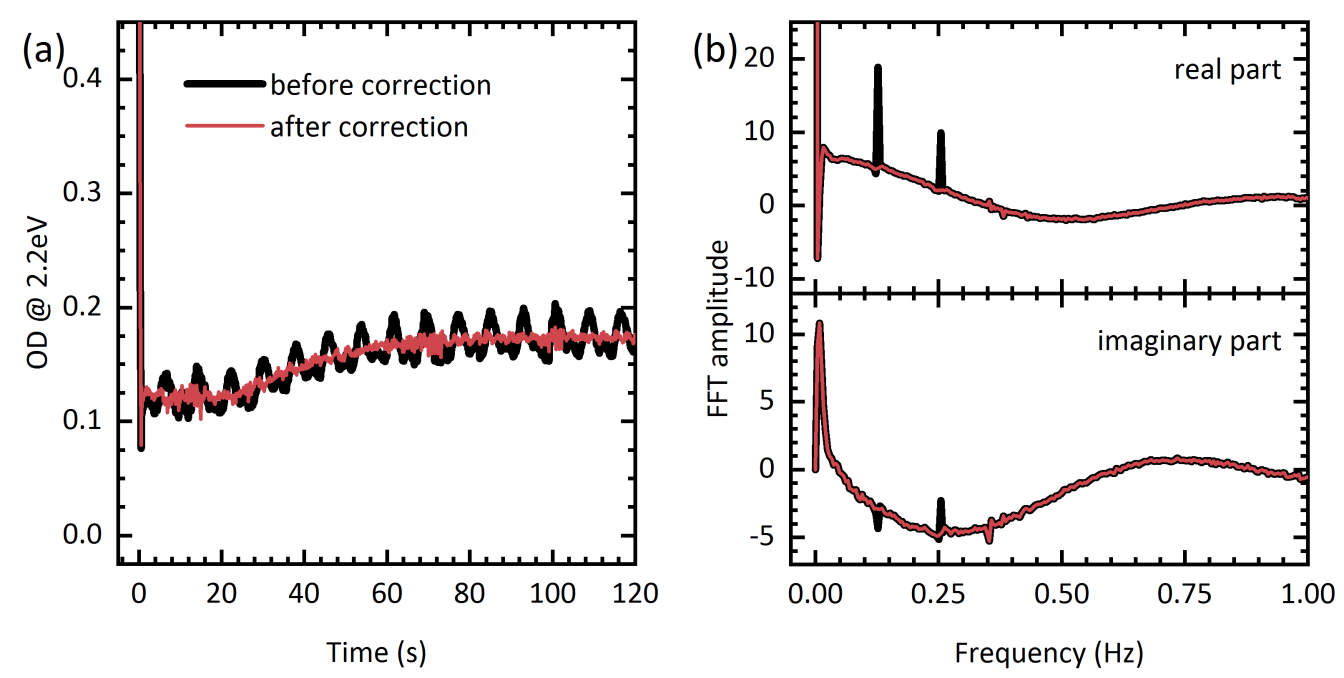

Figure S4: (a) Optical density at $2.2 \mathrm{eV}$ of the TT film from Figure 3 in the main text before and after FFT correction. (b) Corresponding FFT amplitude (top: real part, bottom: imaginary part) before and after correction.

\section{Determination of the fraction of aggregates during processing}

We calculate the fraction of aggregates (FoA) following the approach of Clark et al. ${ }^{5}$, which has been successfully applied to many organic semiconductor materials. $\frac{[6-12]}{-12}$ The basic idea is that the absorption spectrum consists of two separate contributions from amorphous chromophores and aggregated chromophores. It is further assumed that there is a direct transformation from one phase into the other phase. This is indicated by an isosbestic point, which corresponds to the photon energy where the extinction coefficient for both phases is equal.

The fraction of aggregates can be calculated by the fraction of the absorption, which is made up from the aggregated phase after correcting for relative changes in oscillator strength $F$. It can be calculated by the change of aggregate absorption $\Delta A_{\text {agg }}$ divided by the change of amorphous absorption $\Delta A_{\mathrm{am}}$, which is proportional to the ratio of extinction coefficients:

$$
F=-\frac{\Delta A_{\mathrm{agg}}}{\Delta A_{\mathrm{am}}}=\frac{\varepsilon_{\mathrm{agg}}}{\varepsilon_{\mathrm{am}}}
$$

The spectrum of the amorphous film for each spin casting experiment was taken as the first spectrum within the temporal range with an isosbestic point. To suppress noise we averaged the first 10 spectra (about $1.0 \mathrm{~s}$ ), as dynamics are slow. The spectrum of the aggregated species was determined by the scaling method. We averaged the last 20 spectra where film formation was finished and the spectra remained constant. The spectra of the amorphous phase were then multiplied by a factor that they match the high energy side of the final film spectra. The difference between the final film and the scaled amorphous spectrum then yields the spectrum of the aggregated phase. Figure 55 shows this decomposition for TT processed from hexane solution, along with a visualisation of the spectral areas 


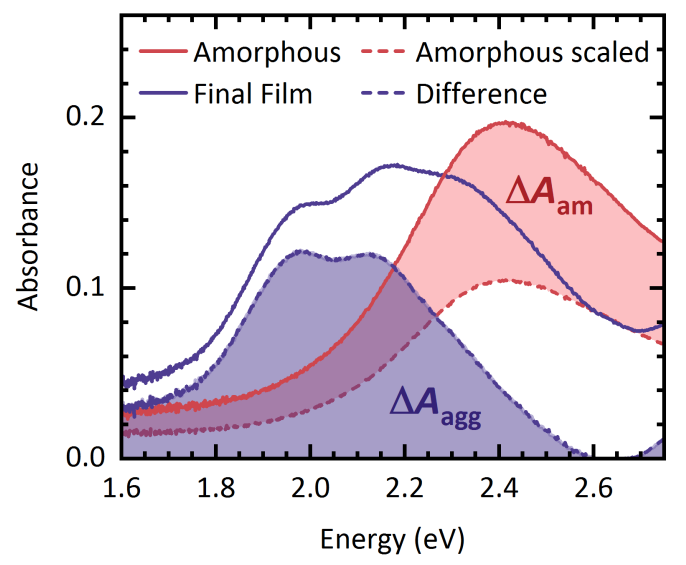

Figure S5: Decomposition of final film absorption into amorphous and aggregated contributions for TT. The spectral areas used for the relative change of oscillator strength are highlighted as well.

needed for the calculation of $F$. This procedure is impossible for the in situ measurement for the CT film. The evolution of the absorption spectra is too fast to allow for reliable calculation of $F$.

The absorption spectra for each single time step were subsequently separated into amorphous and aggregated contributions using a least-square fit for the linear superposition of both spectral components. This allows to calculate an average $F$-value from the corresponding integrals of each time step, yielding $F=0.9 \pm 0.1$. The fraction of aggregates can then be calculated to

$$
\mathrm{FoA}(t)=\frac{A_{\mathrm{agg}}(t)}{A_{\mathrm{agg}}(t)+F \cdot A_{\mathrm{am}}(t)}
$$




\section{References}

[1] Zhou, C.; Cui, Q.; McDowell, C.; Seifrid, M.; Chen, X.; Brédas, J.-L.; Wang, M.; Huang, F.; Bazan, G. C. Topological Transformation of $\pi$-Conjugated Molecules Reduces Resistance to Crystallization. Angew. Chem. 2017, 129, 9446-9449.

[2] Buchhorn, M.; Wedler, S.; Panzer, F. Setup to Study the in Situ Evolution of Both Photoluminescence and Absorption during the Processing of Organic or Hybrid Semiconductors. J. Phys. Chem. A 2018, 122, 9115-9122.

[3] Crameri, F. Geodynamic Diagnostics, Scientific Visualisation and StagLab 3.0. Geosci. Model Dev. 2018, 11, 2541-2562.

[4] Wedler, S.; Bourdick, A.; Athanasopoulos, S.; Gekle, S.; Panzer, F.; McDowell, C.; Nguyen, T.-Q.; Bazan, G. C.; Köhler, A. What Is the Role of Planarity and Torsional Freedom for Aggregation in a $\pi$-Conjugated Donor-Acceptor Model Oligomer? J. Mater. Chem. C 2020, 8, 4944-4955.

[5] Clark, J.; Chang, J.-F.; Spano, F. C.; Friend, R. H.; Silva, C. Determining Exciton Bandwidth and Film Microstructure in Polythiophene Films Using Linear Absorption Spectroscopy. Appl. Phys. Lett. 2009, 94, 163306.

[6] Panzer, F.; Bässler, H.; Köhler, A. Temperature Induced Order-Disorder Transition in Solutions of Conjugated Polymers Probed by Optical Spectroscopy. J. Phys. Chem. Lett. 2017, 8, 114-125.

[7] Perevedentsev, A.; Chander, N.; Kim, J.-S.; Bradley, D. D. C. Spectroscopic Properties of Poly(9,9Dioctylfluorene) Thin Films Possessing Varied Fractions of $\beta$-Phase Chain Segments: Enhanced Photoluminescence Efficiency via Conformation Structuring. J. Polym. Sci. Pol. Phys. 2016, 54, 1995-2006.

[8] Reichenberger, M.; Love, J. A.; Rudnick, A.; Bagnich, S.; Panzer, F.; Stradomska, A.; Bazan, G. C.; Nguyen, T.-Q.; Köhler, A. The Effect of Intermolecular Interaction on Excited States in p DTS(FBTTH2)2. J. Chem. Phys. 2016, 144, 074904.

[9] Reichenberger, M.; Baderschneider, S.; Kroh, D.; Grauf, S.; Köhler, J.; Hildner, R.; Köhler, A. Watching Paint Dry: The Impact of Diiodooctane on the Kinetics of Aggregate Formation in Thin Films of Poly(3-Hexylthiophene). Macromolecules 2016, 49, 6420-6430.

[10] Scharsich, C.; Lohwasser, R. H.; Sommer, M.; Asawapirom, U.; Scherf, U.; Thelakkat, M.; Neher, D.; Köhler, A. Control of Aggregate Formation in Poly(3-Hexylthiophene) by Solvent, Molecular Weight, and Synthetic Method. J. Polym. Sci. Pol. Phys. 2012, 50, 442-453.

[11] Scharsich, C.; Fischer, F. S. U.; Wilma, K.; Hildner, R.; Ludwigs, S.; Köhler, A. Revealing Structure Formation in PCPDTBT by Optical Spectroscopy. J. Polym. Sci. Pol. Phys. 2015, 53, 1416-1430.

[12] Unger, T.; Panzer, F.; Consani, C.; Koch, F.; Brixner, T.; Bässler, H.; Köhler, A. Ultrafast Energy Transfer between Disordered and Highly Planarized Chains of Poly[2-Methoxy-5-(2-Ethylhexyloxy)1,4-Phenylenevinylene] (MEH-PPV). ACS Macro Lett. 2015, 4, 412-416. 